\title{
Block Error Detection Driven Nonlinearity Compensation for Optical Fiber Communications
}

Yankov, Metodi Plamenov; Mehmood, Tayyab; Iqbal, Shajeel; Forchhammer, Søren; Porto da Silva, Edson

Published in:

IEEE Photonics Technology Letters

Link to article, DOI:

10.1109/LPT.2021.3068491

Publication date:

2021

Document Version

Publisher's PDF, also known as Version of record

Link back to DTU Orbit

Citation (APA):

Yankov, M. P., Mehmood, T., Iqbal, S., Forchhammer, S., \& Porto da Silva, E. (2021). Block Error Detection Driven Nonlinearity Compensation for Optical Fiber Communications. IEEE Photonics Technology Letters, 33(9), 461-64. https://doi.org/10.1109/LPT.2021.3068491

\section{General rights}

Copyright and moral rights for the publications made accessible in the public portal are retained by the authors and/or other copyright owners and it is a condition of accessing publications that users recognise and abide by the legal requirements associated with these rights.

- Users may download and print one copy of any publication from the public portal for the purpose of private study or research.

- You may not further distribute the material or use it for any profit-making activity or commercial gain

- You may freely distribute the URL identifying the publication in the public portal 


\title{
Block Error Detection Driven Nonlinearity Compensation for Optical Fiber Communications
}

\author{
Metodi P. Yankov ${ }^{\circledR}$, Member, IEEE, Tayyab Mehmood ${ }^{\circledR}$, Graduate Student Member, IEEE, Shajeel Iqbal ${ }^{\circledR}$, \\ Søren Forchhammer ${ }^{\circledR}$, Member, IEEE, and Edson Porto da Silva ${ }^{\circledR}$, Senior Member, IEEE
}

\begin{abstract}
The perturbation-based nonlinearity compensation (NLC) method is considered in conjunction with a forward error correction (FEC) code that is capable of detecting block errors. The FEC decoding is performed prior to the NLC, and NLC is then performed only for blocks, that could not be decoded correctly. The total NLC complexity is thus dramatically reduced to down to $1 \%$ in some cases w.r.t. the standard, hard decision-directed NLC performed prior to the FEC decoding. Negligible FEC decoding complexity increase is required for the subsequent decoding of the nonlinearity-compensated blocks. The proposed method achieves and sometimes outperforms the standard perturbation based NLC at a fraction of the overall complexity. Polar codes are used to exemplify the method.
\end{abstract}

Index Terms-Nonlinear compensation, fiber optic communications, error-detection, FEC.

\section{INTRODUCTION}

$\mathbf{N}$ ONLINEARITY compensation (NLC) has been a hot topic in the optical fiber research community for more than a decade as a tool to improve the performance of wavelength division multiplexed (WDM) communication systems [1]. Digital back-propagation is known to be effective for compensation of the in-band nonlinearities, but at a significant computational complexity and latency cost. To that end, the perturbation based (PB) NLC has been proposed [2], which operates at one sample per symbol and does not require fast Fourier transforms to be performed. The approach requires estimates of the transmitted symbols (typically through hard symbol decision by applying a slicer) in order to estimate the amplitude and phase nonlinearities accumulated during transmission. At high symbol error rate (SER), the symbol estimates can be erroneous and degrade the overall PB NLC

Manuscript received March 17, 2021; accepted March 21, 2021. Date of publication March 26, 2021; date of current version March 31, 2021. This work was supported in part by the Danish National Research Foundation (DNRF) Research Centre of Excellence, Silicon Photonics for Optical Communications (SPOC) under Grant DNRF123, in part by the Innovation Fund Denmark (IFD) under Grant 9066-00027A, in part by the IFD INnovative solutions for next generation COMmunications infrastructure (INCOM) under Grant 8057-00059B, and in part by the National Council for Scientific and Technological Development (CNPq), Brazil under Grant 432214/2018-6. (Corresponding author: Metodi P. Yankov.)

Metodi P. Yankov, Tayyab Mehmood, Shajeel Iqbal, and Søren Forchhammer are with the Department of Photonics Engineering, Technical University of Denmark, $2800 \mathrm{Kgs}$. Lyngby, Denmark (e-mail: meya@fotonik.dtu.dk).

Edson Porto da Silva is with the Department of Electrical Engineering, Federal University of Campina Grande (UFCG), Campina Grande 58429-900, Brazil.

Color versions of one or more figures in this letter are available at https://doi.org/10.1109/LPT.2021.3068491.

Digital Object Identifier 10.1109/LPT.2021.3068491 performance. This can be circumvented by employing an iterative forward error correction (FEC) and PB NLC equalization [3], in which case the FEC reduces the SER at each iteration and thus improves the PB NLC performance. This approach requires significant complexity and latency increase.

In this letter, the complexity of the FEC-assisted PB NLC is addressed by employing a concatenated soft-decision (SD) and hard-decision (HD) FECs, similar to the emerging 400ZR scheme [4] or the legacy OTN standard [5]. The fact that the SD inner FEC can be error-detecting is exploited in order to select the subset of symbols requiring NLC, while bypassing NLC for symbols, already corrected by the SD FEC. This results in significantly reducing the complexity of the $\mathrm{PB}$ NLC, while only marginally increasing the overall FEC complexity and maintaining the performance of the conventional perturbation-based NLC.

\section{SySTEM SETUP}

The proposed transmitter is given in Fig. 1. Data are encoded by an outer HD FEC, bit-interleaved and encoded by an inner SD FEC. The bits are then mapped to the desired modulation format, symbol interleaved and sent on the channel. In this letter, the HD FEC is considered fixed and not included in the simulation. An interleaver of sufficient length is assumed between the SD and HD FEC, such that the errors are uniformly distributed after the SD FEC decoder. Without loss of generality, the performance is measured in terms of the bit error rate (BER) out of the SD decoder. The BER is then a good predictor of the performance of the HD decoder [6] and thus the overall concatenated FEC system performance.

\section{A. Proposed FEC}

The transmitter is inline with the OIF 400ZR implementation agreement, where a Hamming code of a relatively high fixed rate is used for SD FEC. In this letter, we apply the polar code (PC) proposed as a replacement in [7]. The PC is decoded using cyclic redundancy check (CRC) aided successive cancellation list decoding with a list size of 32 and a CRC length of 16 . The rate of the PC, and thus the overall rate can be easily controlled with nearly continuous granularity by selecting the number $K$ of information bits encoded by the code. In this letter, the code length $N=1024$. A 64QAM modulation and four code rates are considered with $K=700,800,900,1000$ resulting in data rates of $\eta=4.01,4.59,5.18$ and 5.77 bits/QAM symbol (excluding the overhead of the outer FEC). The CRC bits are part of the remaining $N-K$ bits in the codeword. 


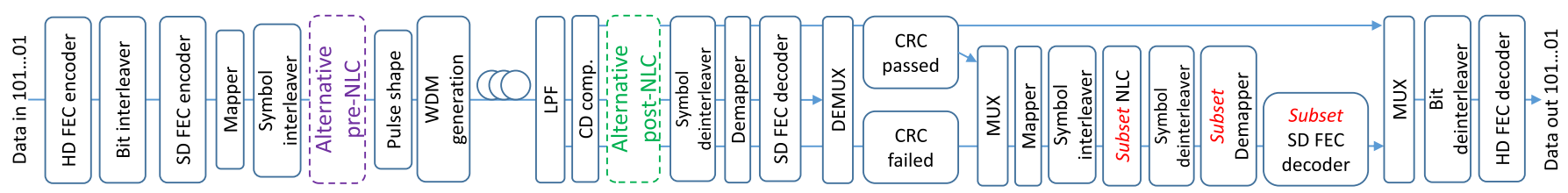

Fig. 1. Proposed symbol interleaved architecture. Outer HD FEC is not simulated. The alternative reference NLC schemes are shown in the dashed blocks.

\section{B. Perturbation-Based NLC}

The PB NLC applied in this work relies on modeling fiber propagation of a single modulated channel through the additive-multiplicative model

$$
\hat{A}_{x}(k)=\left(A_{x}(k)+\Delta A_{x}(k)\right) \exp \left(j \phi_{x}(k)\right)+n_{x}(k),
$$

where $A_{x}(k)$ and $\hat{A}_{x}(k)$ are the transmitted and received symbols at time $k$ on polarizations ' $\mathrm{X}$ ', respectively, $n_{x}(k)$ is a Gaussian noise term, $\Delta A_{x}(k)$ is the nonlinear amplitude noise term and $\phi_{x}(k)$ is the nonlinear phase noise term. The nonlinear terms are given in [3, Sec. II-A], and are also provided here in Eq. (2) and Eq. (3) for completeness (and the complexity analysis in Section III). In Eq. (2) and Eq. (3), $C(m, n)$ is a matrix of mixing coefficients, which describes the crosstalk from symbols at time separation of $\pm m, \pm n$ symbol periods and is calculated as in [2]. The model for polarization ' $Y$ ' follows in a similar manner. The length $L$ should be long enough to capture the total dispersion on the link, and is in practice limited to the nonnegligible terms of the matrix $C$. In this letter, $L=80$, and the number of triplet terms that have to be calculated is $(2 \cdot L+1)^{2}$.

$$
\begin{aligned}
\Delta A_{x}(k) & \sum_{\substack{m \in[-L ; L] \backslash 0 \\
n \in[-L ; L] \backslash 0}} A_{x}(k-n) A_{x}^{*}(k-m+n) A_{x}(k-m) C(m, n) \\
& +\sum_{\substack{m \in[-L ; L] \backslash 0 \\
n \in[-L ; L] \backslash 0}} A_{y}(k-n) A_{y}^{*}(k-m+n) A_{x}(k-m) C(m, n) \\
& +\sum_{\substack{m \in[-L ; L] \backslash 0 \\
n \in[-L ; L]}} A_{y}(k-n) A_{y}^{*}(k-m+n) A_{x}(k-m) C(m, n) .
\end{aligned}
$$$$
\phi_{x}(k)
$$$$
\begin{aligned}
& \propto \operatorname{Im}\left[\left(2\left|A_{x}(0)\right|^{2}+\left|A_{y}(0)\right|^{2}\right) C(0,0)\right] \\
& +\operatorname{Im}\left[\sum _ { m \in [ - L ; L ] \backslash 0 } \left(2\left|A_{x}(k-m)\right|^{2}\right.\right. \\
& \left.\left.\quad+\left|A_{y}(k-m)\right|^{2}\right) C(m, 0)\right] .
\end{aligned}
$$

An estimate of the transmitted sequence is obtained at the receiver and applied to (1) instead of the true symbols in order to calculate an estimate of the intra-channel nonlinear terms, which are then subtracted from the received sequence to obtain a sequence with equalized intra-channel nonlinearities and thus a higher overall SNR (inter-channel nonlinearities are treated as noise). Alternatively, NLC can be performed at the transmitter by using the true transmitted symbols [8], which however alters the transmitted waveform, and thus has an effect both on the effectiveness of the NLC and potentially on the implementation penalties at the transmitter [9].

\section{Proposed and Reference System Architectures}

The proposed receiver architecture is denoted errordetection directed (EDD) NLC and is detailed in Fig. 1. After low-pass filtering, chromatic dispersion compensation, downsampling to one sample per symbol, deinterleaving and demapping using a Gaussian auxiliary channel [10], the SD FEC decoding is applied. The decoded sequence is split into two branches for the codewords that passed and failed the CRC, respectively. In the case of non-CRC-aided FEC, their corresponding error-detection method is applied, e.g. a multiplication with the parity check matrix in the case of the 400ZR-defined expurgated Hamming code or block LDPC codes. The NLC is applied only to the symbols, corresponding to the codewords where the CRC failed in order to improve their SNR. Demapping and a second stage of SD FEC decoding is then applied to those codewords. The resulting decoded codewords are then multiplexed with the codewords that passed CRC during the original decoding, bitdeinterleaved and sent to the outer HD decoder (not simulated in this work).

The proposed method is compared to pre-NLC (at the transmitter side) [8] and post-NLC (at the receiver side) using $\mathrm{HD}$ on the received symbols to drive the calculations in Eq. (1-3) [3]. Since there is no way of assessing at the transmitter which symbols will be erroneous after transmission, the fiber nonlinearity must be calculated for all symbols in the sequence in either case and the two approaches thus have the same complexity. The key benefit in this letter is to calculate the perturbation-based fiber nonlinearity only at the symbols of interest (those that did not pass CRC), and use it to improve detection of those symbols only.

\section{Results AND COMPlEXity ANALYSIS}

A dual polarization, five channel WDM system is simulated using the split-step Fourier method with a step size of $0.1 \mathrm{~km}$ and oversampling factor of 32 . The channels are modulated at $32 \mathrm{GBd}$ with $37.5 \mathrm{GHz}$ grid spacing, and ideal lasers are assumed at the transmitter and receiver. Standard single mode fiber parameters are considered with loss $\alpha=0.2 \mathrm{~dB} / \mathrm{km}$, dispersion $D=17 \mathrm{ps} /(\mathrm{nm} \cdot \mathrm{km})$, nonlinear coefficient $\gamma=$ $1.3 \mathrm{1} /(\mathrm{W} \cdot \mathrm{km})$ and $80 \mathrm{~km}$ per span. Erbium doped fiber amplification is considered with a noise figure of $5 \mathrm{~dB}$ in every amplifier. A total of 100,000 symbols are transmitted per polarization, resulting in 1170 PC FEC codewords, or 819,000, $936,000,1,053,000$ and 1,170,000 information bits for the four considered data rates, respectively. The performance of the central channel is reported at the optimal launch power 


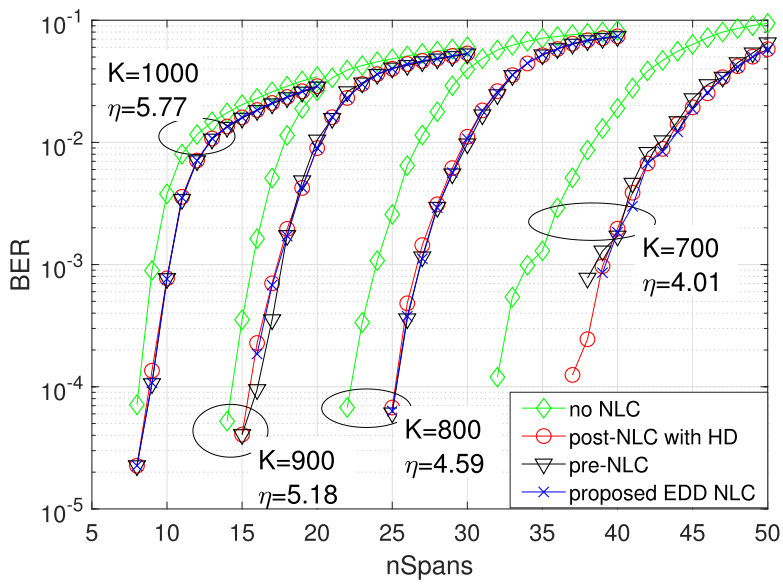

Fig. 2. BER vs. distance for the central channel for the studied NLC schemes.

which is between $-2 \mathrm{dBm}$ and $0 \mathrm{dBm}$ per channel, depending on the distance. The BER for the four considered cases is given in Fig. 2. The proposed scheme performs on par with both reference schemes, and provides a gain of between 1 and 4 spans depending on the distance at a target BER of between $10^{-4}$ and $10^{-2}$.

\section{A. Computational Complexity}

The computational complexity of the nonlinearity calculation is linear with the number of time symbol instants $k$ to be equalized. The computational complexities of the proposed scheme and full NLC can thus be compared directly in terms of NLC and FEC by comparing 1) number of symbols, for which the NLC is applied; and 2) number of codewords decoded twice in the EDD NLC approach. The complexity reduction of the NLC and FEC are first discussed in relative terms, with numbers $>1$ indicating complexity increase and numbers $<1$ indicating complexity reduction.

The resulting complexity is first studied in Fig. 3 as a function of the operating BER (or equivalently, the decoding threshold of the outer FEC). For reference, the thresholds of three popular HD FECs are given: a high-rate (30832, 30592) $\mathrm{BCH}$ code [11] of rate 0.9922, a low-complexity Reed-Solomon code specified in the OTN standard [5] of rate 0.93 and the high-complexity, powerful 400ZR staircase code [4] of rate 0.93 . When the operating BER is low, the number of codewords correctly decoded by the first decoder is increasing, resulting in fewer symbols requiring NLC and at the same time, fewer codewords requiring a second decoding stage. The complexity of the NLC at the BCH code threshold can be reduced by a factor of up to 0.02 , while increasing the complexity of the FEC only by a factor of up to $\approx 1.05$. In the case of the 400ZR threshold, the reduction of complexity in the NLC is reduced to a factor of between $\approx 0.86$ at $K=1000$ and $\approx 0.6$ at $K=700$ due to the higher operating threshold and thus higher block error rate after the first decoding stage. At the same time, $\approx 50 \%$ (a factor of 1.5 ) increase in complexity of the FEC is observed, especially for high PC rates. In general, it appears that lower code rates provide lower NLC complexity. This may be attributed to the

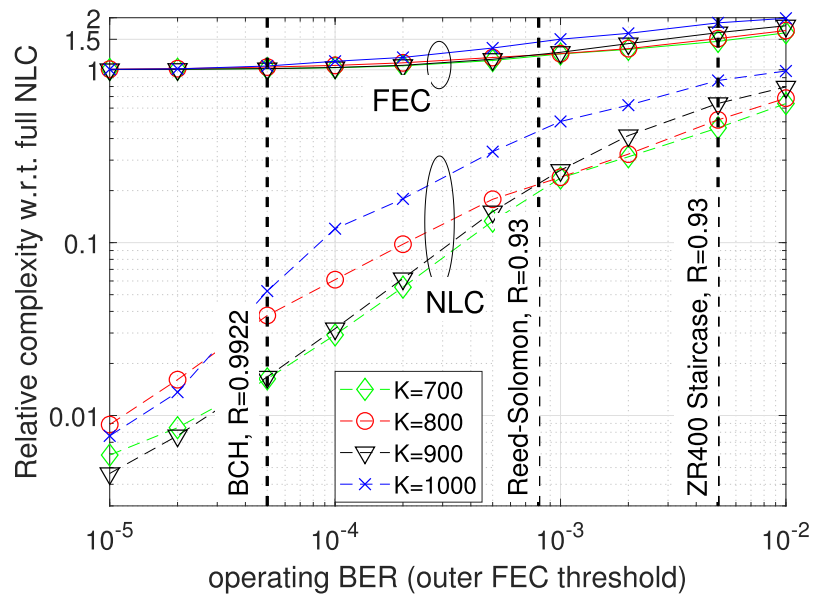

Fig. 3. Relative complexity of the FEC and NLC blocks of the proposed scheme w.r.t. full NLC as a function of the operating BER. The decoding thresholds of three example FECs are exemplified.

fact that the $\mathrm{PC}$ is more efficient at those rates, resulting in better error correction relative to the high-rate conditions and thus fewer symbols requiring NLC.

Estimating the net complexity savings in absolute terms instead of relative terms is challenging without relying on an actual implementation e.g. in an FPGA or ASIC. The problem can be approached by comparing the absolute complexities of the NLC and FEC functions in terms of the basic operations required by each function. The complexity of a polar code parameterized similar to this work with a list size of 32 , similar rates and a codelength of $K=1024$ (which is slightly longer than the maximum considered $K=1000$ and thus represents a worse case scenario in terms of complexity) can be estimated to $\approx 2 \cdot 10^{6}$ additions, selections and multiplexing operations [12, Fig. 6]. We assume those operations have similar implementation complexity. The PB NLC requires $7 \cdot(2 \cdot L)^{2}+2 \cdot 3 \cdot(2 \cdot L+1)$ complex-valued multiplications and $3 \cdot(2 \cdot L)^{2}+2 \cdot(2 \cdot L+1)$ complex-valued additions (see Eq. (2) and Eq. (3)) per symbol time $k$. Assuming a $v$-bit number representations, each multiplication requires at least $v-1$ adders [13, Ch. 4.7]. In this work, the NLC is parameterized to $L=80$, which for $v=4$ requires a total of $>1.59 \cdot 10^{6}$ real-valued additions (assuming 4 real-valued multiplications per complex-valued multiplication and 2 realvalued additions per 1 complex-valued addition) per symbol $k$, or equivalently, $>272 \cdot 10^{6}$ per codeword for a 64QAM and $N=1024$. The complexity of the receiver is clearly dominated by the NLC. In the worst case scenario considered of 400ZR outer FEC, the FEC complexity is increased by a factor 1.86 , while the NLC complexity is decreased by a factor of 0.86 , which amount to a net complexity saving of a factor of $\approx \frac{\left(0.86 \cdot 272 \cdot 10^{6}+1.86 \cdot 2 \cdot 10^{6}\right)}{272 \cdot 10^{6}+2 \cdot 10^{6}} \approx 0.867$. In the best case scenario of a BCH code at operating threshold of $10^{-5}$ (and similarly, in the case of stand-alone SD FEC), the net complexity saving is of the same order as the NLC saving of a factor of $<0.01$ or $>99 \%$.

\section{B. Interleaver Complexity}

As shown in Fig. 1, an extra symbol interleaving and de-interleaving is required for the proposed setup. 


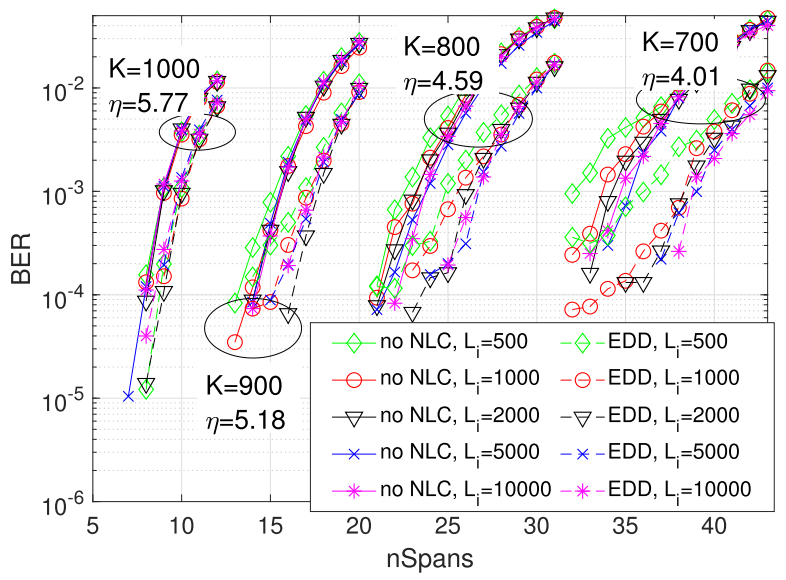

Fig. 4. Interleaver requirements for the proposed scheme.

The performance of the proposed scheme and the corresponding scheme without NLC is given for various interleaver lengths $L_{i}$ in Fig. 4 . We see that $L_{i} \approx 2000$ symbols is sufficient to observe to full benefits of the proposed EDD PB NLC. In perspective, an inter-block interleaver is defined in the Open ZR+ multi-source agreement [14] of length 172.032 bits or 28.672 symbols for 64QAM. Furthermore, the proposed second stage of interleaving/de-interleaving is only performed for a fraction of the blocks (those requiring NLC). We therefore conclude that the added complexity of a second interleaving stage at the receiver is well within the capabilities of modern optical transceivers.

\section{DISCUSSION}

The equalizer length $L$ was increased until the performance benefit of the NLC saturated, which has an obvious relation to the complexity analysis above. In fairness, so were the parameters of the polar code, such as the list size, chosen for optimal performance. Due to the dominating nature of the NLC w.r.t. the FEC block, the net complexity savings are still expected for different parameterization of those blocks. Furthermore, the complexity of other state of the art SD blocks, such as turbo and LDPC for the chosen code length are similar to the studied polar code (see [12, Fig. 6]), with thereby similar expected net savings from the proposed method.

We note that in [3], the turbo equalization achieves the dataaided NLC performance due to the much longer codeword $(N=64800)$ and multiple iterations. Such a powerful SD FEC is not in the scope of the considered 400ZR-style lowcomplexity SD FEC. Furthermore, the proposed architecture is feed forward and thus latency is improved w.r.t. turbo equalization in addition to complexity. We also note that the performance of the EDD NLC can be marginally increased by considering further successive NLC and decoding iterations on codewords with failed CRC, but at unjustifiable increase in complexity and latency.

The proposed scheme is applicable with any modulation format and any SD FEC code that provides error detection. This includes the 400ZR-defined expurgated Hamming code.
However, its rate is too high to allow for long distance transmission and thus see significant NLC performance benefits. At the same time, as seen from the complexity analysis above, high rates typically result in reduced NLC complexity savings due to the reduced SER, and might not be sufficient to offset the FEC complexity increase in this case.

Finally, we note that the proposed EDD NLC is not constrained to concatenated FEC and can be applied to systems with stand-alone error-detection capable FEC with benefits left for future work to confirm.

\section{CONCLUSION}

Concatenated FEC assisted nonlinearity compensation (NLC) was proposed. The fact that the soft decision inner FEC is error detecting was exploited for pruning symbols which do not require NLC in order to in some cases dramatically reduce the NLC complexity. This comes at marginal complexity increase of the FEC in most cases of interest. Performance gains of up to 4 spans were demonstrated w.r.t. conventional receiver architecture without NLC at an overall fraction of complexity w.r.t. the standard perturbation based NLC.

\section{REFERENCES}

[1] J. C. Cartledge, F. P. Guiomar, F. R. Kschischang, G. Liga, and M. P. Yankov, "Digital signal processing for fiber nonlinearities [invited]," Opt. Exp., vol. 25, no. 3, pp. 1916-1936, Feb. 2017.

[2] Z. Tao, L. Dou, W. Yan, L. Li, T. Hoshida, and J. C. Rasmussen, "Multiplier-free intrachannel nonlinearity compensating algorithm operating at symbol rate," J. Lightw. Technol., vol. 29, no. 17, pp. 2570-2576, Sep. 1, 2011.

[3] E. P. D. Silva, M. P. Yankov, F. D. Ros, T. Morioka, and L. K. Oxenlowe, "Perturbation-based FEC-assisted iterative nonlinearity compensation for WDM systems," J. Lightw. Technol., vol. 37, no. 3, pp. 875-881, Feb. 1, 2019.

[4] Implementation Agreement 400ZR, IA 0.10-Draft, Standard, OIF Opt. Internetworking Forum, 2020.

[5] Forward Error Correction for High Bit-Rate DWDM Submarine Systems, Standard Recommendation G.975.1, ITU-T, 2004.

[6] A. Alvarado, E. Agrell, D. Lavery, R. Maher, and P. Bayvel, "Replacing the soft-decision FEC limit paradigm in the design of optical communication systems," J. Lightw. Technol., vol. 34, no. 2, pp. 707-721, Jan. 15, 2016

[7] T. Mehmood, M. P. Yankov, A. Fisker, K. Gormsen, and S. Forchhammer, "Rate-adaptive concatenated polar-staircase codes for data center interconnects," in Proc. Opt. Fiber Commun. Conf. Exhib. (OFC), 2020, p. Th1I.6.

[8] A. Ghazisaeidi and R.-J. Essiambre, "Calculation of coefficients of perturbative nonlinear pre-compensation for Nyquist pulses," in Proc. Eur. Conf. Opt. Commun. (ECOC), Sep. 2014, pp. 1-3.

[9] T. Oyama, T. Hoshida, H. Nakashima, C. Ohshima, Z. Tao, and J. C. Rasmussen, "Impact of pulse shaping and transceiver electrical bandwidths on nonlinear compensated transmission," in Proc. Opt. Fiber Commun. Conf. Exhib. (OFC), 2013, p. OTh3C.2.

[10] M. P. Yankov et al., "Nonlinear phase noise compensation in experimental WDM systems with 256QAM," J. Lightw. Technol., vol. 35, no. 8, pp. 1438-1443, Apr. 15, 2017.

[11] D. S. Millar et al., "Design of a $1 \mathrm{~Tb} / \mathrm{s}$ superchannel coherent receiver," J. Lightw. Technol., vol. 34, no. 6, pp. 1453-1463, Mar. 15, 2016.

[12] S. Shao et al., "Survey of turbo, LDPC, and polar decoder ASIC implementations," IEEE Commun. Surveys Tuts., vol. 21, no. 3, pp. 2309-2333, 3rd Quart., 2019.

[13] M. M. Mano and M. D. Cilleti, Digital Design, 5th ed. London, U.K.: Pearson, 2013.

[14] Open ZR+ Multi-Source Agreement: Technical Specification, Rev. 1.0, Standard, OIF Opt. Internetworking Forum, Sep. 2020. 